\title{
Pre- and Postnatal Influences of Neurohormonal Triggering and Behaviour on the Immune System of Offspring
}

\author{
Laura Yvette Gorczynski ${ }^{\mathrm{a}}$, Christopher Paul Gorczynski ${ }^{\mathrm{a}}$, Tulay Terzioglu ${ }^{\mathrm{b}}$ and Reginald Gorczynski ${ }^{\mathrm{a}, *}$ \\ ${ }^{\mathrm{a}}$ University Health Network and Toronto Hospital, Toronto, ON, Canada \\ ${ }^{\mathrm{b}}$ Akdeniz University, Antalya, Turkey
}

\begin{abstract}
It has been realized for some time that exposure of the developing neonate from conception through birth to (toxic) environmental agents can affect the subsequent health and well-being of the offspring. While organogenesis (the first eight weeks of human gestation) is recognized to be a highly susceptible period for the induction of malformations, the fetal/neonatal developmental phases are now acknowledged to be just as sensitive for many well-documented developmental deficits, amongst which are immune-related disorders. In addition, developing predominantly from studies of maternal stress during pregnancy, a growing body of literature now exists to show that changes in the neurohormonal milieu of both mother/infant during pregnancy and weaning can change immune potential of the offspring. Perhaps even more provocative, there is data to suggest that immune changes in the pregnant mother can in turn modify behavior in offspring. The review below provides a framework for consideration of many of the factors contributing to such processes, and their possible mechanism(s) of action.
\end{abstract}

Keywords: Feto-maternal interactions, stress, immunity, behaviour, neurohormones

\section{INTRODUCTION}

There is a large and growing literature describing how immune responses are influenced by other systems within the individual, in particular the central nervous system [1]. Amongst these constraints there is evidence for direct anatomical interaction between immune cells and nervous tissue [2]; for a crucial role for soluble factors (neurotransmitters; neurohormones; cytokines) which allow "cross-talk" between the two systems [3-5]; and even for an effect of "behaviour", in its broadest sense, on the ability to respond to immunological stimuli $[6,7]$. In the latter context typical demonstrations of the latter come from studies showing a relationship between depression, or

*Correspondence to: Dr. R. Gorczynski, Rm.2-805, MaRS Tower, 101 College Street, Toronto, M5G1L7, ON, Canada. Tel.: +416-581-7519; Fax: +416-581-7515; E-mail: rgorczynski@ uhnres.utoronto.ca. depressed mood, and (lower) immune responses [8,9]. Our own group's interest in this area was sparked by evidence that many immune responses in rodents were clearly subject to regulation by a classical conditioning paradigm described elsewhere by Ader and Cohen [6]. Having successfully shown that antibody responses in mice could be studied in this manner [10], we were particularly intrigued by subsequent observations that the ability to achieve this regulation with populations of mice was itself apparently a function of the intrinsic behaviour shown by the mice before conditioning regimes were applied. Even more provocative was the evidence that this behavior (of offspring, in terms of "conditionability") was in turn predicted to a large degree by behaviour seen within their mothers (and/or foster mothers prior to weaning) [11]. Such observations were consistent with evidence elsewhere suggesting that there may be an imprinting effect of behavioural changes during gestation in mothers on factors which might be predicted to 
affect the subsequent immune responses (or immune response potential) of their offspring [12]. The review that follows will discuss the studies which have supported such a hypothesis, and outline in brief some of the mechanism(s) suggested to account for such observations.

\section{MATERNAL STRESS AND ALTERED IMMUNITY IN OFFSPRING}

The idea that maternal prenatal stress is hazardous to infant health is not a new one. Alterations to maternal hormonal and immune function following stress may be anticipated to impact on both the immune function and neurodevelopment of the fetus. Indeed, a review by Ruiz and Avant [13] considered that prenatal stress in the mother could produce lasting effects on both the neurocognitive development of the infant and the development and function of the infant's immune system. Prenatal stress is strongly correlated with depressive symptomatology in the mother (see below) and in a recent review Maes [14] argued forcibly that depression should indeed be considered as an inflammatory disorder. Meta-analysis revealed consistent evidence for elevation of the plasma levels of two cytokines, interleukin-(IL)-6 and tumor necrosis factor-alpha (TNF $\alpha)$, synonymous with inflammatorytype disorders with no changes in plasma IL-2 and interferon gamma (IFN $\gamma$ ) levels. Moreover, additional elevations in neopterin, sTNFR-1 or sTNFR-2 levels and induction of indoleamine 2, 3-dioxygenase (IDO) with lowered levels of plasma tryptophan and increased levels of tryptophan catabolites along the IDO pathway were commonly associated with depression. Analysis of patients receiving interferon-alpha (IFN $\alpha$ )-based immunotherapy showed that baseline and IFN $\alpha$-induced activation of T cells, IDO activity were related to the development of depressive symptoms [14].

\section{Human studies}

Maternal stress (along with maternal urogenital tract infection) is independently known to be a crucial factor in premature delivery, currently the most important problem in maternal-child health in the United States. Both factors are more prevalent in the population of sociodemographically disadvantaged women known to be at increased risk for preterm birth. Nevertheless, not all women with high levels of stress and/or infection deliver preterm, and currently we do not know what factors modulate susceptibility to pathophysiological events which regulate the endocrine and immune systems in pregnancy. It has been speculated that maternal stress may affect a neuroendocrine pathway, resulting in premature and/or a greater degree of activation of the maternal-placental-fetal endocrine systems to promote parturition. Alternatively, or in addition, such stress may act via an immune/inflammatory pathway, to modulate characteristics of systemic and local (placental-decidual) immunity, thus altering susceptibility to intrauterine and fetal infectious-inflammatory processes and promoting parturition through proinflammatory mechanisms [15]. Given the fact that neuroendocrine and immune processes are extensively cross-regulated, it is to be expected that simultaneous exposure to both high levels of chronic stress and infectious pathogens in pregnancy would produce an interactive effect to produce increased risk for preterm birth. Such effects might produce, indirectly, even greater effects on immune system development in the offspring.

Stress itself is known to be triggered by multiple unexpected environmental stimuli, including aggressive behavior, fear, forced physical activity, sudden environmental changes, social isolation or pathological conditions. Following stressful experiences during early life, such as might be expected following maternal stress during fetal ontogeny, it has been reported that permanent changes in the responsiveness of the nervous system may be observed (programming or imprinting). Such programming effects are thought to be primarily mediated at the level of the hypothalamic-pituitary-adrenocortical (HPA) axis, brain neurotransmitter systems, sympathetic nervous system, and/or the cognitive abilities of the offspring. All of these, in independent studies, have been shown to alter neural regulation of immune function. The affect of prenatal or early life stress on both early immune system development, and on maladaptive immune responses to stress that occur later in life, must be considered. Indeed, altered neural-immune interactions may represent an indirect route by which immunotoxicants affect the developing immune system, and they may indeed do so in a developmentally-restricted manner [16].

More recently Mattes et al. [17] reported on the relationship between maternal depression (as assessed by the Beck Depression Inventory (BDI)) in the second trimester of a cohort of 83 females and measurements of neonatal immune function performed using cord blood. Their results found that $\sim 22 \%$ 
of women manifested mild to moderate depressive symptoms $(\mathrm{BDI}>$ or $=10)$, and that lymphoproliferative responses to a range of stimuli (including egg ovalbumin and cat allergen) was increased in the cord blood of neonates born to these women compared with neonates born to women with normal BDI scores $(<10)$. The former group also showed higher spontaneous cytokine production (IL-6 and IL-10) and higher stimulated cytokine responses to both bacterial antigens and allergens. These data were independent of maternal age and education, parity, gestation, infant gender, and delivery method.

\section{Studies in experimental rodents}

Because of the greater ease of performing controlled experiments in inbred laboratory rodents, a relatively larger body of literature is available from studies with these animals which have explored the importance of maternal stressors during pregnancy on immune responses of offspring. In one such study in rats, stress was applied in utero in the form of prenatal exposure to loud noise (a fire-alarm bell at $\sim 90$-decibles from day 15-21 gestation), and the development and responsiveness of in vitro and in vivo humoral and cellular immune function studied in the offspring [18]. Age-dependent and mitogen-specific decreases in lymphoproliferative activity and reduced immunoglobulin $\mathrm{G}$ levels were observed by postnatal day 21 , along with reductions in titers to herpes simplex virus type 1, and diminished delayed-type hypersensitivity reactions to BSA and adjuvant-induced inflammation. In an attempt to explore whether maternal glucocorticoids, and more specifically dehydroepiandrosterone (DHEA), a weak androgen that is also secreted in response to stress, might be responsible for such alterations in immune responses in offspring, pregnant rats were treated with different doses of DHEA in the drinking water from day 8 of gestation until parturition. This prenatal DHEA treatment reduced the mitogen response to Concanavalin A (ConA) in spleen cells of prepubertal males only, along with increased anterior pituitary expression of pro-opiomelanocortin (POMC) mRNA [19].The existence of sexual dimorphism in the response of offspring to maternal stress was commented upon also by Viveros et al. [20] who observed that maternal deprivation stress produced greater effects on the endocannabinoid system (hippocampal endocannabinoid levels and receptors) in male offspring than females, with the most pronounced effects observed at an early neonatal age (postnatal day13), likely attributable to organizational effects of gonadal steroids. Both the elevated corticosterone and decreased leptin levels observed in animals with maternal deprivation correlated with the deficit in their immunological system [20].

In a study using restraint stress in pregnant mothers, again significant alterations of in vitro and in vivo cellular and humoral immunity of male progeny was seen in offspring of rats at 6 months, with little to no affect observed at an earlier age (7 weeks) [21]. In this case immune perturbations were observed as increased circulating $\mathrm{CD}^{+}{ }^{+}$and natural killer (NK) cells along with evidence for increased IFN $\gamma$ mRNA expression. In vitro stimulation with phytohaemagglutinin (PHA) also resulted in an increased proliferation response of $\mathrm{T}$ lymphocytes and secretion of IFN $\gamma$ using cells from offspring of restrained mothers.

Interesting observations confirming an effect of maternal stress on immunity in offspring have come from studies in mice in which pregnant animals received daily footshock $(0.2-0.4 \mathrm{~mA})$ from day $15-19$ of gestation. Male offspring were studied at 2 months, with assessment of a number of immunological parameters, as well as parameters which reflected altered behaviour. Palermo-Neto et al. [22] reported a decreased locomotor activity in the open-field central zone along with a decreased number of entries into the open arms of the plus-maze and decreased time spent in the exploration of those arms when using mice born to stressed mothers in comparison to controls. In addition, there was decreased macrophage spreading and phagocytosis without concomitant changes in production of reactive nitrogen species from macrophages, along with a diminished ability to restrain the growth of the transplantable Ehrlich ascites tumor. In a somewhat similar study reported later, Fonseca et al. [23] reproduced many of these observations, and reported in addition that the offspring of mothers subject to maternal stress responded differently to a subsequent footshock stress delivered at 30 or 60 days postnatally. The changes were most evident in terms of an effect of shock on altered macrophage spreading and nitric oxide (NO) production, both constitutive and PMA-induced. Both studies concluded that these data were best understood in terms of the perturbation of neuroimmune interactions involving catecholaminergic pathways.

\section{Studies in other species and non-human primates}

There are several points of interest evolving from studies in other experimental animal species which likely have applicability to man. It has been appreciated 
for some time that the reliance of young vertebrates on innate immunity and an associated inflammatory response often leads to reduced growth rates after antigenic challenge. An exception occurs in scenarios where the offspring have inherited maternal antibodies that recognize the antigen, in which case blockade of stimulation of the inflammatory response could be anticipated to attenuate affects on growth suppression. A study to test these hypotheses in female Japanese quail and their newly hatched chicks was described by Grindstaff et al. [24], in which mothers were immunized with lipopolysaccharide (LPS), killed avian reovirus vaccine, or with a placebo (saline). In general, immunization decreased growth in the offspring except where the offspring were immunized with the same antigen as their mother, when enhanced growth in comparison to siblings immunized with a different antigen was observed. The authors concluded that the growth suppressive effects of antigen exposure during development was partially ameliorated by maternal antibodies, but in their absence the offspring were more dependent on innate immune defenses which caused growth retardation.

Considerable interest has developed over the past decade in the role of Microbiota and control of development of intestinal $\mathrm{T}$ cell immune responses. Germ-free mice are known to have poorly developed intestinal immune systems, which can be regenerated if repopulated with mouse (but not human) bacterial species. Interestingly, there is $<10 \%$ overlap in human/mouse gut bacterial flora (by species), and it seems that control of development of intestinal immunity lies chiefly with segmented filamentous bacteria [25]. The failure to reconstitute murine immunity with human bacterial species has been investigated by Kasper's group in some detail, and they have reported that cross-species (bacterial) repopulation with equivalent bacterial loads resulted in poor development of $\mathrm{CD}^{+} \mathrm{T}$ cells in the intestinal epithelial lymph (IEL), Peyer's Patch (PP) and mesenteric lymph node (MLN) compartments. Differences were seen even with repopulation (within mice) using flora from different species, which may be correlated with disease susceptibility [26]. This issue has been further explored by Sonnenburg's group, who have studied the manner in which the "signature" of intestinal flora, selected for fermentation of high fibre foods (e.g. complex glycans from plants) is changed in the context of altered diets. Thus Gnotobiotic mice repopulated with B.Theta and at least one other bacterial strain, receiving a high inulin load, selectively expand B.Theta, which are able to digest this particular glycan, at the expense of alternate species. No such selective expansion occurred on a normal mouse diet [27]. To date it remains unknown if concomitant changes in immunity occur. These studies suggest that in future we may consider characterization of individuals in terms of their microbiome, and that selective alteration of this (and thus the immune system) by dietary changes may be an alternative approach to diseases of the intestine, and perhaps even systemic disorders [27]. These issues take on added importance with the evidence (below) that the development of intestinal immune function is regulated by fetomaternal interactions.

In addition to the microbiome, which is but one environmental factor regulating gut immunity, the development of normal mucosal immune function is now known to be profoundly influenced by maternal and behavioral factors, with the impact of these factors greatest during the prenatal and immediately postnatal periods, but extending beyond this period to development in postnatal life. Husband and Gleeson [28] has discussed age-related changes occurring in mucosal-associated lymphoid tissue in a variety of animal models and in humans, and how such changes were potentially influenced by interactions between the immune and neuroendocrine systems. Included in this discussion were effects mediated by prenatal and postnatal stressors, feeding patterns, nutritional factors, infections, and exposure to allergens and toxins. In the context of such work, the effect of restraint stress delivered to pregnant sows in their last five gestational weeks on the development and reactivity of the immune system of their offspring, studied by Tuchscherer et al. [29], takes on great interest. Maternal stress produced a reduction in serum $\mathrm{IgG}$ in suckling piglets at 1 and 3 days of age, and reduced proliferation in response to the $\mathrm{T}$ cell mitogen concanavalin A (ConA) in cells harvested from infants at postnatal days 1 and 7. NK cell cytotoxicity was not altered by prenatal stress, and there were relatively less consistent effects on B cell mitogen-induced proliferation. Morbidity and mortality during the suckling period was increased in prenatally stressed litters, as shown by a higher frequency of diseased and dead piglets per litter. Furthermore, prenatal stressed caused an altered response to corticotropin (ACTH) injection in piglets compared to non-stressed controls, consistent with the hypothesis that gestational stress in pigs affected the ontogeny of the fetal immune system with both consequences on the susceptibility to diseases (short-term) and the immune responsiveness to stressful stimuli of the offspring at later times. It would be of interest to explore the potential role of an altered microbiome 
in the offspring of maternally stressed individuals to assess if and how this might be causally implicated in the changes seen.

With a view to uncovering effects of maternal stress on immunity in offspring in non-human primates, infant monkeys born after normal or disturbed pregnancies were used as donors of cells for mixed lymphocyte cultures (MLCs), aimed at assessing the infants' proliferative responses to mitomycin-treated stimulator cells either from a genetically unrelated animal or from a virally transformed monkey cell line. Periods of daily stress for 6 weeks in mid-late pregnancy (months 3.0-4.5) produced a significant decrease in proliferative responses, whereas the same stressor early in pregnancy (months 1.5-3.0) increased the responses observed using the neonate's cells. Inhibition of proliferative responses by neonatal cells was independently induced by dexamethasone administered for 2 days late in pregnancy, at 4.5 months following conception and 1 month before term, somewhat analogous to the "late stress" effects seen. Coe et al. [30] concluded from these studies that some immune responses at birth were extremely sensitive to prior prenatal events, while the bidirectional changes also suggested that there may be critical periods in gestation when the same extrinsic events have radically different effects on the fetus. The same group also investigated placental transfer of maternal antibody in either control neonatal squirrel monkeys (63) or animals born to mothers undergoing either a single or 3 periods of disturbance $(21$ or 29 animals respectively). Levels of IgG were determined in mothers and neonates at parturition. Only after a chronic disturbance were altered antibody levels seen in the mothers, resulting in lower IgG levels. Males were born with lower IgG levels, although female infants had higher-than-normal IgG levels despite the lower titers in their mothers. One possible explanation for this effect is that the $\mathrm{IgG}$ receptor may have been selectively up-regulated on placentae of female fetuses thus compensating for the reduced antibody in the disturbed mothers [31]. A subsequent study in rhesus monkeys reinforced these effects of maternal stress on offspring health/immunity. Psychological disturbance and/or pharmacological stimulation of the pituitary-adrenal axis in pregnant females were found to affect the infant's neurological and immune system development. These monkeys had immature neuromotor reflexes at birth, greater emotionality during the first year of life, and a smaller hippocampus as juveniles, along with reduced lymphocyte proliferation, NK activity and cytokine production [32]. Amongst the mechanism(s) implicated in these changes placen- tal transfer of hormones and nutrients were prominent, as indeed (see above) was a significant alteration in microflora in the gastrointestinal tract which was associated with a greater risk for enteric infection.

\section{NON-STRESS-RELATED EFFECTS OF THE PREGNANT MOTHER ON IMMUNITY IN THEIR OFFSPRING}

The communication between the neuroendocrine and immune systems is apparent from a number of phenomena, amongst which are included the well documented "sickness behavior" elicited by cytokines of the immune system that enter the brain to activate second messengers that modify neuronal activity. Such crosstalk between the neuroendocrine and immune systems also leads to production of factors by the nervous and endocrine systems that alter immune cell function and subsequently lead to modulation of immune responses against infectious agents and other pathogens. It is now recognized that neuroendocrine factors likely play a major role in gender-specific differences in the development of certain disorders, including autoimmune/inflammatory diseases that often show a far higher incidence (up to 10-fold) in females compared to males. A recent review by Butts et al. [33] discusses how neuroendocrine factors might modify host immunity to pathogens and contribute to susceptibility/severity of disease development. An interesting example of one such factor, leptin, is discussed in detail by French et al. [34], who commented upon the manner in which this molecule is involved in energy "trade-offs" (between maternal immunity and offspring production) which in turn may modulate the number of offspring reared. The authors build the argument from a consideration that while the primary goal of most organisms is to produce genetic offspring, thus passing their genes on to future generations, nevertheless offspring production is limited by available resources within an environment and so distributing energy among competing physiological systems can in principle result in trade-offs between self-maintenance and offspring investment when resources are limited. In a Siberian hamster model in which the effects of elevated maternal leptin on investment into offspring production versus self maintenance (immune function) was explored, they demonstrated that elevated leptin allowed females to retain more embryos to parturition, and rear more offspring to weaning via reduced maternal infanticide, although this came at a cost of decreased 
innate immune responses in mothers with larger litters [34].

Prolactin (PRL) is a primary lactogenic pituitary hormone which plays a key role in reproduction, from fertilization to mammary gland development and maternal behavior. There are numerous studies too which support a contributory role for PRL in immunoregulation per se, including evidence that hypophysectomized rats suffer from increased thymic atrophy and lymphopenia [35]. Bouchard et al. [36] characterized the development and function of the immune system in PRLR-deficient mice. PRLR-/mice showed no alterations in thymic or splenic cellularity or in the composition of the lymphocyte subsets present in primary (bone marrow and thymus) or secondary (spleen and lymph nodes) lymphoid organs, and lymphocytes from PRLR-/- mice showed normal proliferation to mitogens, cytokines, and allogeneic cells. PRLR-/- splenocytes had normal NK-mediated cytotoxicity as assayed on YAC-1 target cells. More detailed in vivo studies confirmed that PRLR $-/-$ mice generated normal steady-state Ig levels; could mount a normal specific Ig response following immunization with a T-dependent $\mathrm{Ag}$; and could both eliminate allogeneic tumor cells and control Listeria monocytogenes infection. While these data have been interpreted as suggesting that engagement of PRL:PRLR pathways was not crucial to maternal immunomodulation of immunity in offspring, it must be borne in mind that the regulation mediated by the growth and lactogenic family of hormones (HLH), which are closely related and include prolactins, growth hormones and placentral lactogens, all active during pregnancy, is complex. Signaling is mediated by a family of receptors for these hormones which show substantial crossreactivity [37]. As a result, loss of signaling from any one hormone in general is accommodated within this redundant system, with minimal effects observed until ablation of all is achieved. It is indeed now generally accepted that sex hormones, including gonadotropins, prolactin, estrogens, progestogens, androgens and their agonists and antagonists, all play major roles in the neuroimmune regulatory network [38].

A number of groups have established that polyinosinic:polycytidilic (polyI:C)-induced maternal cytokines, particularly IL-6, are involved in the etiology of behavioral abnormalities in their offspring-we will return to this topic in more detail later (see below). At the present time, rather than focus on the abnormal behaviors of offspring born to polyI:C treated mothers, we consider how maternal immune stimulation affects the adaptive immune system of the offspring (see Mandal et al. [39]). This group studied pregnant dams which were stimulated with polyI:C at day 12 of gestation. $24 \mathrm{hrs}$ after stimulation cytokine levels were measured in maternal sera and amniotic fluid. Lymphocytes from offspring were analyzed for Th cell subsets, with the somewhat surprising finding that lymphocytes from offspring of pregnant mothers stimulated with polyI:C developed into Th17 cells following in vitro activation, which was in turn associated with elevated levels of IL-6 in maternal sera and amniotic fluids of polyI:C-injected mothers. In matings between IL-6 "knockout" mothers (IL-6-/-) and wild-type males (IL$6+/+)$ there was no IL- 6 in sera from polyI:C-injected mothers, but high levels of IL-6 was still observed in the amniotic fluids, which was shown to be derived from the fetal (IL-6+/-) component.

Another factor (experienced by the pregnant mother) which has been reported to influence offspring development relates to "social cues" about disease in the maternal environment [40]. Pregnant mice were exposed to infected neighbours by housing them next to animals infected with Babesia microti, and the effect of this indirect immunological exposure on both the females and their adult offspring was examined. Exposed females had higher levels of serum corticosterone compared to females exposed to uninfected neighbours, and produced offspring that, as adults, showed an accelerated immune response to $B$. microt $i$ and less aggression in social groups. These provocative findings led the authors to conclude that ambient information regarding disease is used adaptively to maximize offspring survival and reproductive success in a challenging environment, which clearly has implications for our understanding of epidemiology and individual disease susceptibility. It remains open to speculation that a possible mechanism responsible for these observations may involve transfer from mother to offspring of antibodies and/or cytokines in utero and via the colostrum.

An interesting study has shown that unilateral trauma to the sensorimotor cortex in female rats led to a reduction in the functional activity of NK cells in their offspring. In addition, the offspring showed Tcell immunodeficiency regardless of the lateralization of the trauma in their mothers, although the effect was more severe after right-sided trauma, with both mature and immature T-lymphocytes affected [41]. The mechanism(s) behind these observations remain unexplored.

Before concluding this section, a striking observation by Weaver et al. [12, 42] should be considered. This group found that increased pup licking and 
grooming and arched-back nursing by rat mothers altered the epigenome of offspring at a glucocorticoid receptor gene promoter in the hippocampus. Thus offspring of mothers with the highest levels of both behaviours expressed differences in DNA methylation patterns compared to offspring of mothers without these (or with less pronounced) behaviors within the first week of life. These changes were reversed with cross-fostering, persisted into adulthood and were associated with altered histone acetylation and transcription factor binding to the GR promoter. An infusion of a histone deacetylase inhibitor reversed all of these changes along with the alterations in the HPA axis response to stress. This group concluded that an epigenomic state of a gene could be established through behavioral programming in pregnant mothers, and that it is potentially reversible $[12,42]$.

\section{EARLY NEONATAL STRESS AND ALTERED IMMUNITY}

The potential influence of the pregnant mother on her offspring's immune system extends beyond the period in utero and until weaning. A number of investigators have focused on this period also as being one of critical importance for the developing immune system.

\section{Studies in rodents}

Laban et al. [43] reported on both the effects of noise stress in utero and the stress of early weaning (d15 or 21 rather than 28) on the development and course of experimental allergic encephalomyelitis (EAE) in rats. All animal offspring were immunized with guinea pig spinal cord in complete Freund's adjuvant at 8 weeks of age and clinical signs of EAE were recorded. Following stress in utero they observed suppressed neurological and histological signs of EAE in offspring compared with controls, while in contrast, animals which were weaned early developed more severe clinical signs and histological lesions of disease when compared with controls. In a different model design, but still exploring the effect of neonatal stress on the developing immune system, Spencer et al. [44] subjected male rats at postnatal days 7, 14, 21 or 28 to either saline or LPS injection, and explored their response as adults to a further inflammatory challenge with LPS. The febrile and cyclooxygenase- 2 responses to LPS were attenuated in rats given LPS at days 14 or 21 , but not in those treated at days 7 or 28 . Additional altered behavioral effects were observed in the offspring of animals receiving LPS challenge during the neonatal period which were not simply explained in terms of differences in maternal attention. A study by Shanks et al. [45] has some relevance in trying to understand the mechanism(s) behind these effects. This group also investigated whether exposure to endotoxin in early neonatal life altered neuroendocrine and immune regulation in adult animals, and found that exposure of neonatal rats to a low dose of endotoxin produced long-term changes in the HPA axis, with elevated plasma corticosterone levels. In addition, they observed increased sensitivity of lymphocytes to stress-induced suppression of proliferation but a protection from adjuvant-induced arthritis, suggesting that early endotoxin exposure programmed changes in the development of both neuroendocrine and immunological regulatory mechanisms.

The effect of neonatal stress on parasitic (Nippostrongylus brasiliensis) infection was explored in rats separated from their mothers for $3 \mathrm{~h}$ daily during postnatal days 2-14, compared to controls which were left undisturbed. At 12 weeks of age, animals were infected with 3000 larvae of $N$. brasiliensis and 7-12 days later the number of intestinal adult worms, fecal egg output, and myeloperoxidase (MPO) activity were measured [46]. Maternal deprivation increased the number of jejunal adult worms and fecal eggs and larvae by 7 days after primary infection, and exacerbated the increase in jejunal MPO activity induced by the infection. At day 12 of infection adult worms were only observed in deprived rats. Despite this attenuated primary response to infection, no loss of resistance was seen to a secondary infection by the same parasite, suggesting that while maternal deprivation facilitated primary infection by $N$. brasiliensis and enhanced the inflammatory response within the jejunum, it did not induce severe breakdown of immunity to $N$. brasiliensis [46]. Similar findings have been made in experimental mice. In one such study, postnatal stress (a 15-min daily exposure to clean bedding for the first 2 weeks of life in the absence of the mother but in the presence of littermates, relative to controls which were unhandled until weaning) resulted in enhanced NK-cell activity and T-cell mitogenesis in mice at 60days of age by comparison with unhandled mice, although B-cell proliferation in response to LPS was unaffected [47]. Infant stimulation has also been shown to alter the disease course in neonatal mice with bacterial infection [48]. Yorty et al. [49] independently asked whether anti-viral immunity postpartum was directly modulated by corticosterone, studying the transmammary transfer of herpes simplex virus (HSV)-specific antibody and the ability of neonates to 
survive HSV-2 infection. They observed that increased postpartum maternal corticosterone reduced the levels of total and HSV-specific IgG in the serum and milk of mothers, and that neonates nursed by these mothers received increased levels of corticosterone, decreased levels of total and HSV-specific IgG and were thus more susceptible to HSV-2-associated mortality. Survival was restored through passive immunization with HSV-specific antibody [49].

It is not known how altered neonatal (mal) nutrition may contribute to metabolic and endocrine changes that in turn modulate immunity. Rats born into one of two groups according to their mothers' diet during lactation (a well-nourished group receiving 23\% of protein, and a malnourished group, receiving $8 \%$ of protein) were subsequently challenged to footshock stress. Plasma corticosterone concentrations, leukocyte counts and humoral immunity were evaluated. Interestingly in the neonatal malnourished animals, footshock-induced stress reduced plasma corticosterone concentration with no changes in the immune parameters evaluated [50]. Another attempt to explore the influence of dietary change during lactation on immunity was made by Belluardo et al. [51] who compared the effects of lactation with milk of different compositions (from different mouse strains in a cross-fostering design) on the development of NK cell activity. Analysis of proteins, lactose, and lipids showed differences among the strains for all components studied and in this study there was a marked strain effect on NK activity and indeed on body weight of young mice. What other effects might play a role in these phenomena? A brief discussion of the potential importance of adipokines in this phenomenon follows.

We have already encountered an effect of leptin on maternally-associated stress effects (see earlier). Independent studies now suggest that both overnutrition and undernutrition can disrupt immunity. Overnutrition increases susceptibility to the development of inflammatory or autoimmune diseases, while starvation or malnutrition is apparently more associated with increased susceptibility to infections. White adipose tissue is thought to play a key role as an endocrine organ producing many active peptides, adipokines, which can regulate immune and inflammatory processes. The adipokines, leptin and adiponectin are key molecules linking nutritional status, metabolism and immunity. Primarily known as a satiety factor regulating body weight by suppression of appetite and stimulation of energy expenditure, it has been reported that serum levels and gene expression of leptin in adipocytes are correlated with the proportion of body fat stores. However leptin is also a pro-inflammatory adipokine inducing $\mathrm{T}$ helper 1 cells, and is thought to contribute to the development and progression of autoimmune responses. Adiponectin is also a key factor, which can act as an insulin-sensitizing adipokine whose production decreases in obesity and in conditions of insulin resistance. Adiponectin has also been reported to serve as an anti-inflammatory factor in regard to atherosclerosis, while in other chronic inflammatory/autoimmune diseases adiponectin may have pro-inflammatory effects since adiponectin production has been correlated with inflammatory markers and disease activity [52]. Leptin is known to play a key role in the regulation of energy balance and neuroendocrine function, as well as being important in regulating a number of physiological systems from immunity to cognition. In adults leptin regulates food intake and energy expenditure primarily via the hypothalamus but it is known that leptin receptors are widely expressed in the developing brain from an early stage, and leptin has profound effects on the proliferation, maintenance, and differentiation of neuronal and glial cells. In the early postnatal period in rodents there is a surge in circulating leptin concentrations yet neonates maintain a high level of food intake, and both feeding behavior and metabolic responses to exogenous leptin administration are absent until weaning, the period during which direct neurotrophic actions of leptin have been demonstrated. At this stage leptin has been shown to promote neurite outgrowth and the establishment of hypothalamic circuitry. It has been speculated that changes in the distribution of hypothalamic leptin receptors during this critical period may underlie the age-specific effects of leptin [53].

\section{Studies in non-human primates}

We conclude this section with a selected sampling of the literature from studies in non-human primates. As could be anticipated there are a number of studies which have related changes seen to parameters discernible in human populations. Differences in the response to maternal separation in nonhuman primate infants have been attributed to the presence or absence of processes that model social support in humans. In this context, alternative attachments to other members of the social group may help buffer the infant against a depressive response to maternal separation. In macaques, by manipulating the presence or absence of other attachment figures during separation it was found that infants who retained such attachments showed fewer behavioral evidences of depression when sep- 
arated from their mothers, and were simultaneously protected from the decreased lymphocyte activation (and NK response) seen in infants without such attachments. These data strongly support the hypothesis that social support, which can accrue from alternative attachments, modulates the response to loss, and can itself thus account for at least some of the individual differences seen in these responses [54].

Following on from considerations of the effect of alterations in the microbiome on the immunity seen in offspring born to stressed mothers, Bailey at al reported on the integrity of the indigenous microflora of the intestines after maternal separation, and its relationship to susceptibilty to pathogen infection [55]. The stability of the indigenous microflora were estimated by evaluating the total and gram-negative aerobic and facultatively anaerobic bacterial species from stool cultures taken before and after maternal separation. This group additionally monitored behavioral and cortisol responses to separation and attempted to correlate these with the microflora. They observed a decrease in fecal bacteria, especially Lactobacilli, by day 3 postseparation, with a return to baseline by the end of the week. The decline in the microflora was correlated with evidence for stress-indicative behaviors, although not with cortisol secretion. Importantly, infants who displayed stress-indicative behaviors were more susceptible to opportunistic bacterial infection consistent with the hypothesis that strong emotional reactions to disruption of the mother-infant bond (post-birth) increased vulnerability to disease [55].

\section{LONGER-TERM EFFECTS OF BEHAVIOUR IN PREGNANCY}

This review evolved from earlier studies from one of the authors which suggested that assessment of maternal behavior might have longer-term implications for immunity in the offpring [11]. It is acknowledged that young vertebrates have only a limited capacity to synthesize antibodies and are dependent on the protection of maternally transmitted antibodies for humoral disease resistance early in life. Nevertheless, this leaves open the possibility that mothers can enhance "fitness" by somehow priming their offspring's immune systems to achieve a state of improved disease resistance. Similar effects of transgenerationally induced defences are well-documented in plants and invertebrates, but there is little evidence for maternal priming of offspring immunity in vertebrates (but see [56, 57]). In a deliberate test of the ability of mothers to stimulate the immune systems of their offspring, Grindstaff et al. [24, 58] manipulated maternal and offspring antigen exposure in a population of wild birds. They showed that immunization of the mother before egg laying stimulated a transgenerational defence against pathogens by elevating endogenous offspring antibody production, which, if the disease environments encountered by mothers and offspring were similar, would presumably act to allow their young to cope better with the local pathogen fauna $[24,58]$.

In another interesting study of the longer-term consequences of deleterious behavior during pregnancy on neonatal immune development and early immune responses Seelig et al. [59] investigated the effect of neonatal exposure to maternally consumed ethanol on the neonates' immune response in adulthood, including immune responses made in the context of additional ethanol exposure. Female rats were fed on either $6 \%$ ethanol or pair-fed isocaloric control liquid diets for 30 days prior to, and during, pregnancy and lactation. One day after weaning their pups, the mothers were infected with 1000 Trichinella spiralis larvae, and maintained on the same diets for a further 20 days, at which time they were challenged with 2000 T. spiralis larvae. All animals were killed 3 days later, and their immune status determined. These animals represented the first generation alcohol animals, while their female offspring represented the experimental second generation animals. These second generation animals also received maternal ethanol exposure during pregnancy and lactation followed by a control diet during their juvenile period (from weaning to 90 days of age), after which they were subjected to a schedule of ethanol or pairfeeding, equivalent to that given to their mothers. There were two groups of second generation animals. In the one, animals were exposed to ethanol during their mother's pregnancy and lactation periods only, but there was no subsequent ethanol treatment. In the second group, animals received ethanol during their mother's pregnancy and lactation periods and this was repeated again throughout their adult experimental period. Previous studies from this same group had suggested perturbed immunity was most evident in secondary immune responses following T.Spiralis challenge. Accordingly this protocol was used to assess the two groups of rats under consideration. No differences between the ethanol and pair-fed groups were observed in intestinal worm burden. Nevertheless, second generation animals receiving alcohol exposure throughout showed reduced proliferation responses to T. spiralis antigen and Con A stimulation relative to the all other groups. This group also produced less specific IgM and IgG anti-T. spiralis antibodies than other 
groups. Thus exposure to maternal ethanol affected immune responses during the second generation of adult life, and maternal alcohol exposure apparently exerted a cumulative immune effect in concert with later consumption of ethanol by offspring born to alcoholic mothers [59].

\section{DO IMMUNE CHANGES IN PREGNANCY AFFECT BEHAVIOUR IN ADULTS?}

In the concluding section we review data which considers not the evidence that behavior, either during or shortly after pregnancy, can modulate immunity in the offspring, but rather the converse, that the immune system (of the mother) during gestation itself provides some influence on subsequent behavior observed in the offspring. Interest in such questions has expanded with the evidence that understanding immunobiology may help us understand in better detail some psychiatric disorders. Amongst the mechanism(s) considered for such effects are included studies on the relationship of cytokine gene expression and psychiatric disorders, with concomitant evidence for activation of the immune system in schizophrenia and depressive disorders [60]. It is known that cytokines, actively transported into the CNS, can activate astrocytes and microglia cells, which in turn can also produce cytokines resulting in a feedback stimulation (and occasionally inhibition) mechanisms. Cytokines influence dopaminergic, noradrenergic, and serotonergic neurotransmission and correlations have been shown between expression of certain cytokines and behaviour, including a relationship between sickness behavior and sleep with IL-1 $\beta$ [7]; of disturbances of memory and cognitive impairment and IL-2, and/or TNF $\alpha$ [61]; and between IL-6 and depressive disorders [62].

Normal pregnancy is associated with an elevated inflammatory state, both systemically in the mother and in the placenta, and there is now evidence that following further inflammatory stimulation, including that associated with maternal infection, there is an enhanced risk of autism and schizophrenia in the offspring. The adult autistic brain and cerebrospinal fluid (CSF) have been shown to have high levels of inflammatory cytokines, and in animal studies maternal immune activation increases the levels of inflammatory cytokines in the fetal environment and in the fetal brain. Accordingly, it has been suggested that maternal immune activation initiates a self-perpetuating cycle of subacute inflammation in the brain which affects both neural development, and also influences ongoing postnatal behavior [63]. The same panel of signaling molecules is now known to be involved in triggering cellular programs initiated by infection (e.g., NF-кB, JAX/STAT, ERK) as are used by the developing brain to orchestrate programs of cell proliferation, differentiation, and migration. Accordingly the potential for crosstalk between the maternal immune response and the developing fetal brain is already "inbuilt", and Smith et al. [64, 65] have hypothesized that this may account in no small way for the evidence that maternal infections are an environmental risk factor for schizophrenia and autism.

As might be expected most of the research literature in this area depends upon experimental manipulations performed in rodents. Ponzio et al. [66] administered daily injections of IL-2 to pregnant mice during mid-gestation (confirming transmission to the fetal embryo by radiolabelling), and analyzed their offspring in comparison to offspring of pregnant mice injected with vehicle only. Offspring to IL-2 treated mothers had multiple immune changes (with elevated splenic immune responses to mitogen and antigen stimulation). Importantly, in addition to increased open-field activity, grooming and rearing behavior, these offspring also displayed new motor learning, namely acquisition of a classically conditioned eyeblink response.

Given the hypothesis, as stated above, that maternal viral infection might be associated with increased risk of abnormal behavior in the offspring, a number of investigators have used animal model systems to explore the potential mechanism(s) involved. Using pregnant mice challenged by respiratory infection (from human influenza virus) it was reported that offspring displayed abnormal behavioral responses as adults, with deficits in the acoustic startle response akin to human autism behaviours [67]. Compared with control animals, offspring of infected mice were deficient in exploratory behavior in both open-field and novelobject tests, deficient in social interaction, and showed distinct responses to administration of antipsychotic (clozapine and chlorpromazine) and psychomimetic (ketamine) drugs. Importantly, it was argued that it was not the pathology induced by the virus itself which caused these changes, since maternal injection of the synthetic double-stranded RNA polyI:C caused similar deficits in the offspring in the absence of virus. It was thus argued that the maternal viral infection which had such a profound effect on the behavior of adult offspring probably produced these effects via an effect of the maternal immune response on the fetus [67]. Zuckerman and Weiner [68] reached a similar 
conclusion after studying infant behavior following treatment of pregnant female mice with polyI:C.

At this juncture then it is pertinent to ask how polyI:C might produce these effects at the molecular level. Disruption of functional and structural dopaminergic development was found in experimental animals in association with the developmental neuropathology associated with psychosis-related abnormalities induced by prenatal exposure to infection [69]. Longitudinal studies in these mice revealed a developmental correspondence between the ontogeny of specific dopaminergic neuropathology and the postnatal onset of several distinct forms of dopamine-dependent functional abnormalities which have been independently implicated in schizophrenia. This same group has thus raised the interesting notion that interventions targeting the developing dopamine system may help prevent, or at least attenuate, the development of psychotic disorders which seem to follow from neurodevelopmental disruption of those same dopamine-dependent functions.

\section{SUMMARY}

It has been argued above that there is now compelling evidence to suggest that a variety of perceived environmental "insults" to pregnant females, and even to nursing females in the post delivery period, in the form of physical, pathogen-related or emotional stressors, can produce significant perturbations in the immune responses seen in their offspring. The mechanisms involved seem to be in part understood in terms of what we know of altered activation of the HPA axis, and of altered cytokine, neurohormone and neutrotransmitter production within the CNS. There is some exciting newer data implying that there may be an evolutionary balance struck between changes in maternal behavior sacrificing some aspects of maternal innate immunity at the expense of improved immunity in offspring. Moreover, more recently we have come to appreciate that even effects as subtle as an altered dietary behavior change in the mother can itself produce profound changes in the microbiome of both mother and offspring, and this also can potentially have important implications for subsequent immune development. With the additional knowledge that these interactions between behavior and immune response potential in mothers and their offspring are reciprocal in nature, so that altered immune activation in the mother may also in turn evoke altered behavior in the offspring, the "loop" becomes closed. As our knowl- edge and understanding in this important area of human development continues to expand we can anticipate that many of the studies discussed above will open up quite novel avenues of approach, including immunological ones, to the analysis and treatment of clinical developmental disorders.

\section{ACKNOWLEDGMENTS}

Supported by: Grants to RG from the Heart and Stroke Foundation of Ontario and CIHR.

\section{REFERENCES}

[1] Ader R, Felten DL, Cohen N. Psychoneuroimmunology, 4th edition, 2 volumes. Academic Press. ISBN 0-12-088576-X; 2006.

[2] Stevens-Felten SY, Bellinger DL. Noradrenergic and peptidergic innervation of lymphoid organs. Chem Immunol. 1997; 69: 99.

[3] Livnat S, Felten SY, Carlson DL, Bellinger FL, Felten DL. Involvement of peripheral and central catecholamine systems in neural-immune interactions. J Neuroimmunol 1985; 10: 5.

[4] Santambrogio L, Benedetti M, Chao MV, Muzaffar R, Kulig K, Gabellini N, Hochwald G. Nerve growth factor production by lymphocytes. J Immunol 1994; 153: 4488.

[5] Grasso G, Massai L, De Leo V, Muscettola M. The effect of LHRH and TRH on human interferon-gamma production in vivo and in vitro. Life Sci. 1998; 62: 2005.

[6] Ader R, Cohen N. Behaviourally conditioned immunsopuppression. Psychosom Med. 1974; 37: 333.

[7] Moldofsky H. Sleep, health and immunocompetence. In: Berczi I, Gorczynski RM, editors. Neuroimmune Biology: New Foundation of Biology. Shannon, Co. Clare, Ireland, Elsevier; 2001.

[8] Kulmatycki KM, Jamali F. Drug disease interactions: role of inflammatory mediators in depression and variability in antidepressant drug response. J Pharm Pharm Sci. 2006; 9: 292.

[9] Blume J, Douglas SD, Evans DL. Immune Suppression and Immune Activation in Depression[Quick Edit]. Brain Behav Immun 2010; in press.

[10] Gorczynski RM. Analysis of lymphocytes in, and host environment of, mice showing conditioned immunosuppression to cyclyophospahmide. Brain Behav Immun. 1987; 1: 21.

[11] Gorczynski RM, Kennedy M. Behavioural trait associated with conditioned immunity. Brain Behav Immun. 1987; 1: 72 .

[12] Weaver ICG, Cervoni N, Champagne FA, D/Alessio AC, Sharma S, Seckl JR, Dymov S, Szyf M, Meaney MJ. Epigenetic programming by maternal behavior. Nature Neuroscience. 2004; 7: 847.

[13] Ruiz RJ, Avant KC. Effects of maternal prenatal stress on infant outcomes: a synthesis of the literature. ANS Adv Nurs Sci. 2005; 28: 345

[14] Maes M. Depression is an inflammatory disease, but cellmediated immune activation is the key component of depression. Prog Neuropsychopharmacol Biol Psychiatry. 2010 Jun 20.

[15] Wadhwa PD, Culhane JF, Rauh V, Barve SS, Hogan V, Sandman CA, Hobel CJ, Chicz-DeMet A, Dunkel-Schetter C, 
Garite TJ, Glynn L. Stress, infection and preterm birth: a biobehavioural perspective. Paediatr Perinat Epidemiol. 2001; 15(Suppl. 2): 17

[16] Bellinger DL, Lubahn C, Lorton D. Maternal and early life stress effects on immune function: relevance to immunotoxicology. J Immunotoxicol. 2008; 5: 419.

[17] Mattes E, McCarthy S, Gong G, van Eekelen JA, Dunstan J, Foster J, Prescott SL. Maternal mood scores in mid-pregnancy are related to aspects of neonatal immune function. Brain Behav Immun. 2009; 23: 380.

[18] Sobrian SK, Vaughn VT, Ashe WK, Markovic B, Djuric V, Jankovic BD. Gestational exposure to loud noise alters the development and postnatal responsiveness of humoral and cellular components of the immune system in offspring. Environ Res. 1997; 73: 227.

[19] Shelat SG, Aird F, Redei E. Exposure to dehydroepiandrosterone in utero affects T-cell function in males only. Neuroimmunomodulation. 1997; 4: 154.

[20] Viveros MP, Llorente R, López-Gallardo M, Suarez J, Bermúdez-Silva F, De la Fuente M, Rodriguez de Fonseca F, Garcia-Segura LM. Sex-dependent alterations in response to maternal deprivation in rats. Psychoneuroendocrinology. 2009; 34(Suppl. 1): S217.

[21] Vanbesien-Mailliot CC, Wolowczuk I, Mairesse J, Viltart O, Delacre M, Khalife J, Chartier-Harlin MC, Maccari S. Prenatal stress has pro-inflammatory consequences on the immune system in adult rats. Psychoneuroendocrinology. 2007; 32: 114.

[22] Palermo Neto J, Massoco CO, aacute F, vare RC. Effects of maternal stress on anxiety levels, macrophage activity, and Ehrlich tumor growth. Neurotoxicol Teratol. 2001; 23: 497.

[23] Fonseca ES, Massoco CO, Palermo-Neto J. Effects of prenatal stress on stress-induced changes in behavior and macrophage activity of mice. Physiol Behav. 2002; 77: 205.

[24] Grindstaff JL, Hasselquist D, Nilsson JK, Sandell M, Smith HG, Stjernman M. Transgenerational priming of immunity: maternal exposure to a bacterial antigen enhances offspring humoral immunity. Proc Biol Sci. 2006; 273: 2551.

[25] Cerf-Bensussan N, Gaboriau-Routhiau V. The immune system and the gut microbiota: friends or foes? Nature Rev Immunol. 2010; 10: 735.

[26] Duan J, Chung H, Troy E, Kasper DL. Microbial colonization drives expansion of IL-1 receptor 1 expressing, IL-17 producing $\gamma / \delta$ T cells. Cell Host \& Microbe. 2010; 7: 140.

[27] Lecuit M, Sonnenburg JL, Cossart P, Gordon JI. Functional genomic studies of the intestinal response to a foodborne enteropathogen in a humanized gnotobiotic mouse model. J. Biol. Chem. 2007; 282: 15065 .

[28] Husband AJ, Gleeson M. Ontogeny of mucosal immunityenvironmental and behavioral influences. Brain Behav Immun. 1996; 10: 188 .

[29] Tuchscherer M, Kanitz E, Otten W, Tuchscherer A. Effects of prenatal stress on cellular and humoral immune responses in neonatal pigs. Vet Immunol Immunopathol. 2002; 86: 195.

[30] Coe CL, Lubach GR, Karaszewski JW. Prenatal stress and immune recognition of self and nonself in the primate neonate. Biol Neonate. 1999; 76: 301.

[31] Coe CL, Crispen HR. Social stress in pregnant squirrel monkeys (Saimiri boliviensis peruviensis) differentially affects placental transfer of maternal antibody to male and female infants. Health Psychol. 2000; 19: 554.

[32] Coe CL, Lubach GR. Prenatal origins of individual variation in behavior and immunity. Neurosci Biobehav Rev. 2005; 29 : 39.
[33] Butts CL, Sternberg EM. Neuroendocrine factors alter host defense by modulating immune function. Cell Immunol. 2008; 252: 7

[34] French SS, Greives TJ, Zysling DA, Chester EM, Demas GE. Leptin increases maternal investment. Proc Biol Sci. 2009; 276: 4003.

[35] Berczi I. The immunology of prolactin. Sem Reprod Endocrinol. 1992; 10: 196.

[36] Bouchard B, Ormandy CJ, Di Santo JP, Kelly PA. Immune system development and function in prolactin receptordeficient mice. J Immunol. 1999; 163: 576.

[37] Berczi I, Szentivanyi A. Growth and lactogenic hormones, insulin-like growth factor and insulin. In: Berczi I, Szentivanyi A, editors. "Neuroimmmune Biology, Volume 3: The Immune-Neuroendocrine Circuitry. History and Progress. Amsterdam: Elsevier; 2003. p. 129-153.

[38] Berczi I, Laatikainen R, Pulkkinen J. Sex Hormones and their Analogues in Neuroimmune Biology. Immun., Endoc. \& Metab. Agents in Med. Chem., 2010; 10: in press.

[39] Mandal M, Marzouk AC, Donnelly R, Ponzio NM. Maternal immune stimulation during pregnancy affects adaptive immunity in offspring to promote development of TH17 cells. Brain Behav Immun 2010; in press.

[40] Curno O, Behnke JM, McElligott AG, Reader T, Barnard CJ. Mothers produce less aggressive sons with altered immunity when there is a threat of disease during pregnancy. Proc Biol Sci. 2009; 276: 1047.

[41] Avaliani TV, Ogurtsov RP, Puzyreva VP, Seryakova OR. Lateralization of brain trauma in female Wistar rats determines the immune and neurological status of offspring. Neurosci Behav Physiol. 2002; 32: 425.

[42] Weaver IC, Meaney MJ, Szyf M. Maternal care effects on the hippocampal transcriptome and anxiety-mediated behaviors in the offspring that are reversible in adulthood. Proc Natl Acad Sci U S A. 2006; 28: 3480.

[43] Laban O, Marković BM, Dimitrijević M, Janković BD. Maternal deprivation and early weaning modulate experimental allergic encephalomyelitis in the rat. Brain Behav Immun. 1995; 9: 9.

[44] Spencer SJ, Martin S, Mouihate A, Pittman QJ. Early-life immune challenge: defining a critical window for effects on adult responses to immune challenge. Neuropsychopharmacology. 2006; 31: 1910.

[45] Shanks N, Windle RJ, Perks PA, Harbuz MS, Jessop DS, Ingram CD, Lightman SL. Early-life exposure to endotoxin alters hypothalamic-pituitary-adrenal function and predisposition to inflammation. Proc Natl Acad Sci U S A. 2000; 97 : 5645 .

[46] Barreau F, de Lahitte JD, Ferrier L, Frexinos J, Bueno L, Fioramonti J. Neonatal maternal deprivation promotes Nippostrongylus brasiliensis infection in adult rats. Brain Behav Immun. 2006; 20: 254.

[47] Neveu PJ, Deleplanque B, Puglisi-Allegra S, D' Amato FR, Cabib S. Influence of early life events on immune reactivity in adult mice. Dev Psychobiol. 1994; 27: 205.

[48] Schlewinski E. Studies on the influence of psychological factors on the immune system: the result of infantile stimulation on the disease course in neonatal mice with bacterial infection Z Psychosom Med Psychoanal. 1975; 21: 390.

[49] Yorty JL, Schultz SA, Bonneau RH. Postpartum maternal corticosterone decreases maternal and neonatal antibody levels and increases the susceptibility of newborn mice to herpes simplex virus-associated mortality. J Neuroimmunol. 2004; 150: 48. 
[50] Barreto-Medeiros J, Queiros-Santos A, Cabral-Filho JE, Ferreira E, Silva WT, Leandro CG, Deiró TC, Manhaes-de-Castro R, Machado Barbosa de-Castro CM. Stress/aggressivenessinduced immune changes are altered in adult rats submitted to neonatal malnutrition. Neuroimmunomodulation. 2007; 14: 229.

[51] Belluardo N, Mudó G, Campisi A, Vanella A, Russo J, Bindoni M. Crossfostering and early development of natural killer cytotoxic activity in various inbred mouse strains. Physiol Behav. 1993; 53: 951.

[52] Stofkova A. Leptin and adiponectin: from energy and metabolic dysbalance to inflammation and autoimmunity. Endocr Regul. 2009; 43: 157.

[53] Cottrell EC, Mercer JG, Ozanne SE. Postnatal development of hypothalamic leptin receptors. Vitam Horm. 2010; 82: 201.

[54] Boccia ML, Scanlan JM, Laudenslager ML, Berger CL, Hijazi AS, Reite ML. Juvenile friends, behavior, and immune responses to separation in bonnet macaque infants. Physiol Behav. 1997; 61: 191.

[55] Bailey MT, Coe CL. Maternal separation disrupts the integrity of the intestinal microflora in infant rhesus monkeys. Dev Psychobiol. 1999; 35: 146.

[56] Gorczynski RM, Kennedy M. A possible maternal effect in the abnormal hyporesponsiveness to specific alloantigens in offspring born to neonatally tolerant fathers. J. Immunol. 1983; 131: 1115 .

[57] Blanden RV, Steele EJ. A unifying hypothesis for the molecular mechanism of somatic mutation and gene conversion in rearranged immunoglobulin variable genes. Immunol. Cell Biol. 1998; 76: 288

[58] Grindstaff JL. Maternal antibodies reduce costs of an immune response during development. J Exp Biol. 2008; 211: 654.

[59] Seelig LL Jr, Steven WM, Stewart GL. Second generation effects of maternal ethanol consumption on immunity to Trichinella spiralis in female rats. Alcohol Alcohol. 1999; 34: 520.

[60] Müller N, Ackenheil M. Psychoneuroimmunology and the cytokine action in the CNS: implications for psychiatric dis- orders. Prog Neuropsychopharmacol Biol Psychiatry. 1998; 22: 1 .

[61] McAfoose J, Baune BT. Evidence for a cytokine model of cognitive function. Neurosci Biobehav Rev. 2009; 33: 355.

[62] Basterzi AD, Aydemir C, Kisa C, Aksaray S, Tuzer V, Yazici K. Göka E. IL-6 levels decrease with SSRI treatment in patients with major depression. Hum Psychopharmacol. 2005; 20: 473 .

[63] Patterson PH, Xu W, Smith SEP, Devarman, BE. Maternal immune activation, cytokines and autism current clinical neurology, 2009; Part IV, 289.

[64] Smith SEP, Patterson PH. Alteration of neurodevelopment and behavior by maternal immune activation. In "The Neuroimmunological Basis of Behavior and Mental Disorders". In: Siegel A, Zalcman SS, editors. Springer Science Publishers; 2009. pp. 111-130.

[65] Smith, SEP, Hsiao, E, Patterson, PH. Activation of the Maternal Immune System as a Risk Factor for Neuropsychiatric Disorders. In "Maternal influences on fetal Neurodevelopment. Clinical and Research aspects". In: Zimmerman AW, Connors SI, editors. Springer Science Publishers; 2010. pp. 97-115.

[66] Ponzio NM, Servatius R, Beck K, Marzouk A, Kreider T. Cytokine levels during pregnancy influence immunological profiles and neurobehavioral patterns of the offspring. Ann N Y Acad Sci. 2007; 1107: 118.

[67] Shi L, Fatemi SH, Sidwell RW, Patterson PH. Maternal influenza infection causes marked behavioral and pharmacological changes in the offspring. J Neurosci. 2003; 23: 297.

[68] Zuckerman L, Weiner I. Maternal immune activation leads to behavioral and pharmacological changes in the adult offspring. J Psychiatr Res. 2005; 39: 311.

[69] Vuillermot S, Weber L, Feldon J, Meyer U. A longitudinal examination of the neurodevelopmental impact of prenatal immune activation in mice reveals primary defects in dopaminergic development relevant to schizophrenia. J Neurosci. 2010; 30: 1270 . 